\title{
Amyloid Fibril Interaction
}

National Cancer Institute

\section{Source}

National Cancer Institute. Amyloid Fibril Interaction. NCI Thesaurus. Code C41565.

Amyloid Fibril Interaction involves temporary non-covalent binding of a molecule through intermolecular physical forces of attraction with insoluble forms of a secretaseprocessed metal ion-binding type I cell surface glycoprotein (APP Family) required for endocytosis and basolateral sorting; important in developing synapses, for cell mobility, adhesion, and axonogenesis; and Notch signaling inhibitor, copper homeostasis regulator, transcription activator, and apoptosis enhancer. 